\title{
Three pathways for trehalose biosynthesis in mycobacteria
}

\author{
Koen A. L. De Smet, ${ }^{1} \dagger$ Anthony Weston, ${ }^{2} \neq$ Ivor N. Brown, ${ }^{1}$ \\ Douglas B. Young ${ }^{1}$ and Brian D. Robertson ${ }^{1}$
}

Author for correspondence: Brian D. Robertson. Tel: +44 207594 3965. Fax: +44 2072626299. e-mail: b.robertson@ic.ac.uk

\footnotetext{
1 Department of Infectious Diseases and Microbiology, Imperial College School of Medicine, St Mary's Campus, Norfolk Place, London W2 1PG, UK

2 Glaxo Wellcome Medicines Research Centre, Stevenage, Hertfordshire SG1 2NY, UK
}

\begin{abstract}
Trehalose is present as a free disaccharide in the cytoplasm of mycobacteria and as a component of cell-wall glycolipids implicated in tissue damage associated with mycobacterial infection. To obtain an overview of trehalose metabolism, we analysed data from the Mycobacterium tuberculosis genome project and identified ORFs with homology to genes encoding enzymes from three trehalose biosynthesis pathways previously characterized in other bacteria. Functional assays using mycobacterial extracts and recombinant enzymes derived from these ORFs demonstrated that mycobacteria can produce trehalose from glucose 6-phosphate and UDP-glucose (the OtsA-OtsB pathway) from glycogen-like $\alpha(1 \rightarrow 4)$-linked glucose polymers (the TreY-TreZ pathway) and from maltose (the TreS pathway). Each of the pathways was found to be active in both rapid-growing Mycobacterium smegmatis and slowgrowing Mycobacterium bovis BCG. The presence of a disrupted treZ gene in Mycobacterium leprae suggests that this pathway is not functional in this organism. The presence of multiple biosynthetic pathways indicates that trehalose plays an important role in mycobacterial physiology.
\end{abstract}

Keywords: genome, mycobacteria, osmotic stress, trehalose, tuberculosis

\section{INTRODUCTION}

Trehalose is a non-reducing disaccharide (1-O- $\alpha$-Dglucopyranosyl- $\alpha$-D-glucopyranoside) found in insects, plants and microbial cells. It has a protective effect on proteins and biological membranes during cryopreservation or desiccation in vitro, and has been implicated in survival of micro-organisms exposed to environmental stresses in vivo. Escherichia coli and Saccharomyces cerevisae contain little or no trehalose during exponential growth under standard culture conditions, for example, but the free sugar rapidly accumulates in response to osmotic stress or heat shock, and during the stationary phase of culture (Strom \& Kaasen, 1993; De Virgilio et al., 1994). In Streptomyces venezuela, trehalose is absent from the mycelium in vegetative cultures, but is abundant in spores (Ranade \& Vining, 1993).

\footnotetext{
Abbreviations: MOT, maltooligosyltrehalose.
}

†Present address: Innogenetics NV, Industriepark Zwijnaarde, 9052 Ghent, Belgium.

$\ddagger$ Present address: Cytocell Ltd., Somerville Court, Banbury Business Park, Adderbury, Oxford OX17 3SN.
In contrast, trehalose is constitutively present in mycobacteria cultured in routine laboratory media; it is the only free sugar readily detectable in the cytoplasm and accounts for $1 \cdot 5-3 \%$ of the total dry weight of the cells (Elbein \& Mitchell, 1973). The function of the free trehalose is unknown. Metabolic studies in the rapidgrowing Mycobacterium smegmatis showed that the trehalose pool is subject to constant turnover, suggesting it is not simply accumulated as an energy reserve (Winder et al., 1972). Trehalose is also incorporated into a range of mycobacterial glycolipids, including cord factor (trehalose 6,6'-dimycolate), sulpholipids (acylated trehalose-2'-sulphate derivatives) and trehalose-containing lipooligosaccharides (Besra \& Chatterjee, 1994). Toxic activities associated with these cell-wall components have led to the suggestion that they may play a role in tissue damage resulting from infection with pathogenic mycobacteria such as Mycobacterium tuberculosis.

The emergence of multidrug-resistant strains of $M$. tuberculosis (Pablos-Mendez et al., 1998) highlights the need to develop novel drugs for treatment of tuberculosis; particularly compounds that could act against the bacterial population that persists during prolonged 
(a) OtsA-OtsB pathway

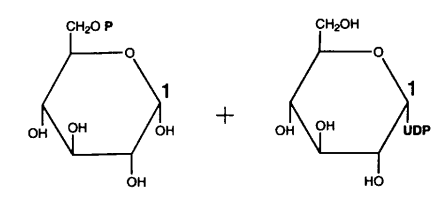

glucose 6-phosphate

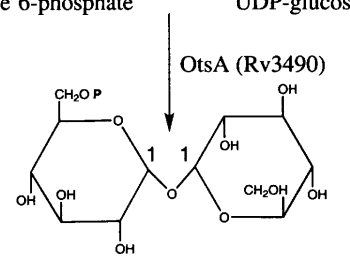

(b) TreY-TreZ pathway

$\alpha(1-4)$ glucose polymers
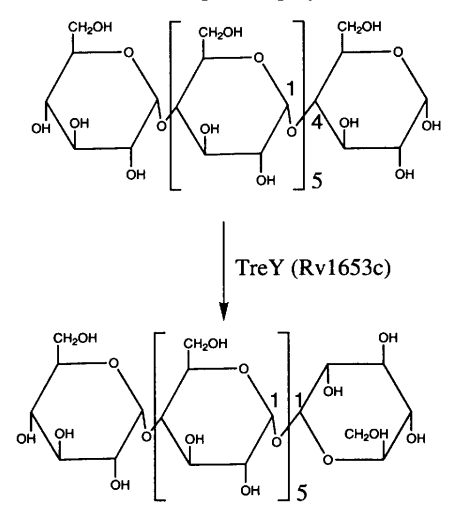

TreZ (Rv1562c)
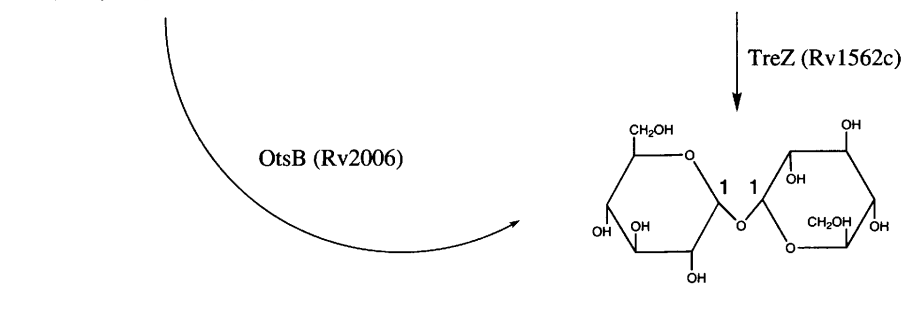

(c) TreS pathway

1-O- $\alpha$-D-glucopyranosyl-6-phosphate- $\alpha$-D-glucopyranoside

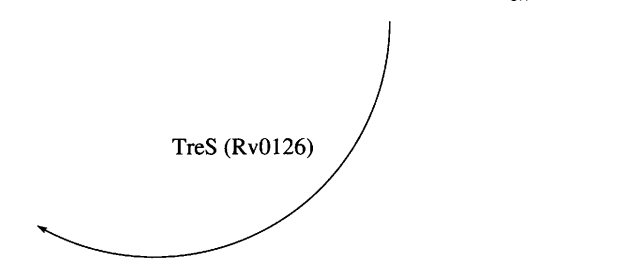

4-O- $\alpha$-D-glucopyranosyl-D-glucose (maltose)

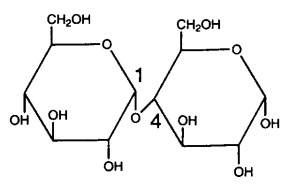

1-O- $\alpha$-D-glucopyranosyl- $\alpha$-D-glucopyranoside (trehalose)

Fig. 1. Trehalose biosynthesis pathways. Three pathways for trehalose biosynthesis have been identified in bacteria. (a) The OtsA-OtsB pathway is found in a wide range of microbes including E. coli and Sac. cerevisiae. (b) The TreY-TreZ pathway is used by Arthrobacter, Rhizobium sp. and Sulfolobus acidocaldarius. (c) The TreS pathway has been characterized in Pimelobacter sp. and in T. aquaticus.

therapy with currently available drugs. The prominence of trehalose in the cytoplasm, and its presence in toxic glycolipids suggests that disruption of trehalose metabolism might represent a useful drug target. Pan et al. (1996) demonstrated that several antibiotics that inhibit growth of $M$. smegmatis had an effect on a trehalose biosynthetic enzyme, and Belisle et al. (1997) demonstrated inhibition of mycobacterial growth and disruption of a trehalose mycolyltransferase enzyme by 6 azido-6-deoxy- $\alpha, \alpha^{\prime}$-trehalose. We have also noticed that trehalosamine (2-amino-2-deoxy- $\alpha$-D-glucopyranosyl $\alpha$ D-glucopyranoside) inhibits the growth of $M$. smegmatis, which may be due to interference with trehalose metabolism. In the present study, we wished to identify enzymes involved in biosynthesis of trehalose in mycobacteria, with the eventual goal of assessing their potential as drug targets.

The best characterized pathway for trehalose biosynthesis involves condensation of glucose 6-phosphate with UDP-glucose to form trehalose 6-phosphate, with subsequent dephosphorylation generating the free disaccharide (Fig. 1a). In E. coli, these reactions are catalysed by trehalose-6-phosphate synthase (the product of the ots A gene) and trehalose-6-phosphate phosphatase (otsB) (Kaasen et al., 1992). Transcription of the $o t s A$ and ots $B$ genes is induced during stationary phase and osmotic stress in E. coli (Kaasen et al., 1994). In Sac. cerevisiae, the two reactions are catalysed by TPS1 and
TPS2, which form a multimeric trehalose synthase complex together with additional subunits, which regulate activation in response to stress (Reinders et al., 1997). An alternative pathway for generation of trehalose from glycogen has been identified in Arthrobacter (Maruta et al., 1996a), Rhizobium (Maruta et al., 1996b) and in the archaeon Sulfolobus acidocaldarius (Maruta et al., 1996c). In this pathway, the terminal $\alpha(1 \rightarrow 4)$ linked residue of the glucose polymer is converted to an $\alpha(1 \rightarrow 1)$ linkage by maltooligosyltrehalose synthase (MOT synthase, encoded by tre Y). The terminal disaccharide is subsequently cleaved by a hydrolase enzyme (MOT trehalohydrolase, encoded by treZ), releasing a free trehalose molecule (Fig. 1b). Finally, a third pathway has been identified in Pimelobacter (Tsusaki et al., 1996) and Thermus aquaticus (Tsusaki et al., 1997), in which the $\alpha(1 \rightarrow 4)$ link in maltose is converted to an $\alpha(1 \rightarrow 1)$ link to form trehalose (Fig. 1c). This reaction is catalysed by trehalose synthase, encoded by the treS gene. Both TreY and TreS are involved in rearranging the attachment at O-4 to O-1, but at least in Pimelobacter (Nishimoto et al., 1996) and Arthrobacter (Nakada et al., 1995) are constrained by the number of additional sugar residues attached to the penultimate glucose.

In mycobacteria, biosynthesis of trehalose from glucose 6-phosphate and UDP-glucose has been demonstrated. Trehalose-6-phosphate synthase (equivalent to OtsA of 
E. coli) has been purified from M. smegmatis, with a molecular mass of 59-60 kDa estimated by gel filtration and PAGE (Pan et al., 1996). Trehalose-6-phosphate phosphatase (OtsB) has also been partially purified, but its molecular mass was not determined (Matula et al., 1971).

The availability of complete bacterial genome sequence information makes it possible to obtain a general overview of metabolic pathways within a particular organism in order to construct a framework for subsequent biological characterization (Moxon, 1995). In the present study, we have screened the genome sequence of M. tuberculosis (Cole et al., 1998) for the presence of ORFs with homology to genes involved in trehalose biosythesis in other bacteria. We report on evidence that all three of the previously described biosynthetic pathways are functional in mycobacteria.

\section{METHODS}

Genomics. M. tuberculosis genome sequence data were analysed using the BLAST server at the Sanger Centre (http://www.sanger.ac.uk/Projects/M_tuberculosis/). Data from the partially completed Mycobacterium leprae genome sequence was similarly searched (http://www.sanger.ac.uk/ Projects/M_leprae/). The identified mycobacterial ORFs were then used to search the non-redundant GenEMBL database with the BLAST2 algorithm through a gateway provided by EMBL (http://www.bork.embl-heidelberg.de:8080/Blast2/), using TBLASTN, the BLOSUM62 matrix and an EXPECT value of 10 , without filtering. Further analysis was performed using the GCG suite of programs sited at the HGMP Resource Centre, Hinxton, UK.

Growth of mycobacteria and preparation of extracts. $M$. smegmatis $\mathrm{mc}^{2} 6$ was grown with shaking at $37^{\circ} \mathrm{C}$ in $\mathrm{LB}$ medium (Gibco BRL). Mycobacterium bovis BCG (Pasteur) was grown in Middlebrook $7 \mathrm{H} 9$ medium with ADC supplement (Difco) and $0.5 \%(\mathrm{v} / \mathrm{v})$ glycerol. Bacteria from exponentially growing cultures $(200 \mathrm{ml})$ were harvested by centrifugation, washed with PBS and resuspended in $1 / 100$ vol. PBS. Cells were disrupted by ultrasonication using a Soniprep 150 (MSE) for $10 \mathrm{~s}$ at an amplitude of $8 \mu \mathrm{m}$, and cell debris was removed by centrifugation for $5 \mathrm{~min}$ in a microfuge. The resulting supernatant was subjected to gel filtration using a PD10 column (Pharmacia) to remove small molecules such as trehalose and other substrates that may interfere with enzyme assays. Protein levels were determined using the Coomassie Plus Protein Assay (Pierce). For routine enzyme assays, cell extracts were used at $0.5 \mathrm{mg}$ protein $\mathrm{ml}^{-1}$. All enzyme assays were performed at least twice using extracts prepared from different cultures, and each assay was perfomed in duplicate.

\section{Assay of trehalose biosynthesis}

Measurement of trehalose. Trehalose was measured by assaying the release of free glucose following incubation of samples with a specific recombinant trehalase from E. coli prepared as described below. Samples for analysis $(10 \mu$ l aliquots $)$ were added to $10 \mu \mathrm{l} 1 \mathrm{M} \mathrm{NaH}_{2} \mathrm{PO}_{4}, \mathrm{pH} 6 \cdot 0,80 \mu \mathrm{l}$ water and $1 \mu \mathrm{l}$ recombinant trehalase in microtitre-plate wells, and incubated at $37^{\circ} \mathrm{C}$ for $1 \mathrm{~h}$. Glucose was then measured by adding $100 \mu \mathrm{l}$ $50 \mathrm{mM} \mathrm{NaH}{ }_{2} \mathrm{PO}_{4}, \mathrm{pH} 6.0$, containing $0.75 \mathrm{mg} \mathrm{o}$-dianisidine $\mathrm{ml}^{-1}, 2 \mathrm{U}$ glucose oxidase and $0 \cdot 1 \mathrm{U}$ peroxidase (all from Sigma). Colour development was measured after $30 \mathrm{~min}$ at $450 \mathrm{~nm}$ using a microtitre-plate reader, and a linear re- lationship was obtained between trehalose concentration $(0 \cdot 2-1 \mathrm{mM})$ and absorbance.

Preparation of recombinant trehalase (TreA). The trehalase enzyme used in the assay was a partially purified preparation of recombinant $E$. coli TreA. The tre A gene, cloned in the expression vector pBAD24, was kindly donated by Michael Ehrmann (University of Konstanz, Germany) together with an E. coli host strain (CS12) defective in several dextrin-cleaving enzymes (Prinz et al., 1996). A periplasmic extract containing the recombinant trehalase was obtained using cold osmotic shock, as described by Nossal \& Heppel (1966). Although other proteins were present in the recombinant preparation, no glucose was produced from maltose, maltoheptaose, sucrose, fructose, galactose or glycogen.

Trehalose biosynthesis assay. The assay mixture consisted of mycobacterial extract $(40 \mu \mathrm{l})$ with appropriate substrates in a final volume of $100 \mu \mathrm{l}$ in $100 \mathrm{mM} \mathrm{NaH}_{2} \mathrm{PO}_{4}$ at $\mathrm{pH} 6 \cdot 0$. For the OtsA-OtsB pathway, the substrates were UDP-glucose $(10 \mathrm{mM})$ and glucose 6-phosphate $(5 \mathrm{mM})$, with $10 \mathrm{mM}$ $\mathrm{MgCl}_{2}$. For the TreY-TreZ pathway, maltoheptaose (Sigma) was added at a concentration of $1 \%(\mathrm{w} / \mathrm{v})$. For the TreS pathway, maltose (Sigma) was added at $1 \%(\mathrm{w} / \mathrm{v})$. Assays were incubated at $37^{\circ} \mathrm{C}$ for up to $1 \mathrm{~h}$ and the reaction stopped by boiling for $10 \mathrm{~min}$. Denatured protein was removed by centrifugation for $2 \mathrm{~min}$ in a microfuge and aliquots of the supernatant were used to determine the level of trehalose using the assay described above.

\section{Other enzyme assays}

Trehalose-6-phosphate synthase. Trehalose-6-phosphate synthase (OtsA) activity was measured by determining the release of UDP from UDP-glucose in the presence of glucose 6phosphate. Mycobacterial cell extract or purification fraction was added to a final volume of $100 \mu \mathrm{l}$ in $20 \mathrm{mM}$ Tris $/ \mathrm{HCl}, \mathrm{pH}$ $7 \cdot 5$, containing $10 \mathrm{mM}$ glucose 6 -phosphate, $5 \mathrm{mM}$ UDPglucose, $0.04 \mathrm{mg}$ heparin $\mathrm{ml}^{-1}$ (which enhances the enzyme activity; Pan et al., 1996) and $10 \mathrm{mM} \mathrm{MgCl}_{2}$, and incubated at $37^{\circ} \mathrm{C}$ for $20 \mathrm{~min}$. The sample was then boiled for $5 \mathrm{~min}$, denatured proteins removed by centrifugation, and the amount of UDP in the supernatant measured in terms of oxidation of NADH in a linked assay with pyruvate kinase and lactic acid dehydrogenase. The assay was carried out in microtitre-plate wells containing $2.5 \mathrm{mM}$ phosphoenol pyruvate, $0.5 \mathrm{mM} \mathrm{NADH}, 10 \mathrm{mM} \mathrm{MgCl}, 50 \mathrm{mM}$ Tris $/ \mathrm{HCl}, \mathrm{pH}$ 7.5, $3 \mathrm{U}$ pyruvate kinase $\mathrm{ml}^{-1}$ and $3 \mathrm{U}$ lactic acid dehydrogenase $\mathrm{ml}^{-1}$ (both from Sigma). The decrease in absorbance at $340 \mathrm{~nm}$ was measured continuously in a microtitre-plate reader over a period of $20 \mathrm{~min}$.

Trehalose-6-phosphate phosphatase. Phosphatase activity was measured in a final volume of $50 \mu \mathrm{l}$ containing $10 \mathrm{mM}$ Tris/ $\mathrm{HCl}, \mathrm{pH} 7 \cdot 0,2 \mathrm{mM} \mathrm{MgCl}_{2}, 2 \mathrm{mM}$ trehalose 6-phosphate (Sigma) and an appropriate amount of enzyme preparation, in a microtitre plate at $37^{\circ} \mathrm{C}$ for $0-30 \mathrm{~min}$. To this, $200 \mu \mathrm{l}$ filtered $1 \%$ ammonium molybdate, $0.15 \%$ malachite green and $12.5 \%(\mathrm{v} / \mathrm{v})$ concentrated $\mathrm{HCl}$ was added and the absorbance at $630 \mathrm{~nm}$ measured to determine the amount of free phosphate produced (Matula et al., 1971).

Maltose synthesis. Conversion of trehalose to maltose by $M$. tuberculosis trehalose synthase (TreS) was measured in an assay format analogous to that used to measure trehalose biosynthesis. Trehalose $(1 \%, \mathrm{w} / \mathrm{v})$ replaced maltose as the substrate, and glucose release was measured following incubation with maltase (Sigma) in place of trehalase.

Purification and $\mathrm{N}$-terminal sequencing of $\boldsymbol{M}$. smegmatis OtsA. Trehalose-6-phosphate synthase (OtsA) was purified 
Table 1. Sequence of primers used in this study

\begin{tabular}{|ll|}
\hline Name & \multicolumn{1}{c|}{ Sequence $\left(\mathbf{5}^{\prime}-\mathbf{3}^{\prime}\right)$} \\
\hline OtsA-F & AAAAGGATCCATGACTTCGCGGGGTAAC \\
OtsA-R & GATCTTAAGCTTATGTCGCGTCGCGCGC \\
OtsB-F & AAGGATCCATGCCGGTCACCATAGAG \\
OtsB-Fcore & AAGGATCCATGTCGGAACTTCCCGAC \\
OtsB-R & AAAAGCTTGCGTTGAGTGTCACTAAG \\
TreS-F & TTGCTGATCAGTGCAGGGCAGTCACGTCGAA \\
TreS-R & TTGCAAGCTTGTCATAGGCGCCGCTCTCCC \\
TreY-F & AAGGATCCATGGCATTTCCGGTTATTTCC \\
TreY-R & AGCTAAGCTTTCAGGCATTGTCGCGCAC \\
TreZ-F & AGCTAAGCTTTCAGGCCGGCTCCGCAGC \\
TreZ-R & CGGGATCCATGCCTGAATTCCGAGTATGGG \\
\hline
\end{tabular}

from $M$. smegmatis using a procedure adapted from that described by Pan et al. (1978). M. smegmatis cell paste $(25 \mathrm{~g}$ wet weight) was suspended in $30 \mathrm{ml} 50 \mathrm{mM}$ Tris/ $\mathrm{HCl}$ buffer, $\mathrm{pH} 7 \cdot 5$, and lysed by sonication. Intact cells and insoluble debris were removed by centrifugation. Protein was precipitated in $50 \%$ saturated ammonium sulphate, collected by centrifugation, dissolved in $8 \mathrm{ml} 50 \mathrm{mM}$ Tris/ $\mathrm{HCl}, \mathrm{pH} 7 \cdot 5$, and dialysed overnight against the same buffer. The sample was fractionated by ion-exchange chromatography on a Mono Q-Sepharose column using a gradient of $0-500 \mathrm{mM} \mathrm{KCl}$ by HPLC. Fractions with enzyme activity were pooled and concentrated by $70 \%$ saturated ammonium sulphate precipitation and dialysis. The product was further fractionated on a heparin-agarose affinity column using a $50-1000 \mathrm{mM}$ $\mathrm{KCl}$ gradient. All fractions were analysed for enzyme activity and by SDS-PAGE and Coomassie staining. Only those positive for trehalose-6-phosphate synthase activity were found to contain a band of $56 \mathrm{kDa}$. The positive fractions were pooled, separated by Tricine gel electrophoresis, blotted onto a PVDF membrane, the $56 \mathrm{kDa}$ protein was eluted and $\mathrm{N}$-terminal sequence determined on an automated ABI 476A protein sequencer (Applied Biosystems).

\section{Production of recombinant proteins}

Histidine-tagged recombinants. ORFs and partial ORFs with homology to ots $A$, ots $B$, tre $\mathrm{Y}$, tre $\mathrm{Z}$ and treS genes were amplified from $M$. tuberculosis and M. leprae chromosomal DNA by PCR using Deep Vent DNA polymerase (New England Biolabs) and the primers shown in Table 1.

The PCR products were cloned either directly into the expression vector $\mathrm{pQE30}$ (Qiagen), or into the vector pPCRScript-SK (Stratagene), prior to subcloning into pQE30. Recombinant clones were grown and expression induced by addition of IPTG according to the manufacturer's recommendations. In the case of the TreS construct, a fraction of the recombinant protein was found to be soluble in the bacterial lysate. This was purified from a $1 \mathrm{ml}$ cleared sonicate obtained from an induced $100 \mathrm{ml}$ culture, using an Ni-NTA spin column (Qiagen) according to the manufacturer's instructions. For the remaining clones, insoluble protein products were denatured with $8 \mathrm{M}$ urea and purified by affinity chromatography under denaturing conditions on a Ni-NTA column, according to the manufacturer's recommended conditions (Qiagen). Purified recombinant proteins were renatured by stepwise dialysis against $50 \mathrm{mM}$ Tris $/ \mathrm{HCl}(\mathrm{pH} 8 \cdot 0)$ solutions containing $5 \mathrm{M}, 2.5 \mathrm{M}, 1 \mathrm{M}$ and no urea, followed by another dialysis against $50 \mathrm{mM}$ Tris $/ \mathrm{HCl}, \mathrm{pH} 8 \cdot 0$.
Recombinants in $M$. smegmatis. For expression in recombinant mycobacterial systems, PCR products containing the $M$. tuberculosis tre $Y$ and tre $Z$ genes were cloned in the vector pACE (De Smet et al., 1999). This is based on the mycobacterial-E. coli shuttle vector pOLY-G with the addition of a $3 \mathrm{~kb}$ fragment from the region upstream of the acetamidase gene of M. smegmatis (Parish \& Stoker, 1997). Recombinant M. smegmatis clones were grown in minimal medium with $0 \cdot 2 \%(\mathrm{w} / \mathrm{v})$ succinate as the carbon source and $50 \mu \mathrm{g}$ hygromycin $\mathrm{ml}^{-1}$ (Boehringer). For protein expression, acetamide $(0 \cdot 2 \%, \mathrm{w} / \mathrm{v})$ was added to exponential cultures which were grown for a further $24 \mathrm{~h}$ prior to harvesting and preparation of extracts by sonication.

\section{RESULTS}

\section{Analysis of the M. tuberculosis genome sequence}

Sequences generated from the M. tuberculosis genome project were screened for the presence of ORFs sharing homology with genes encoding components of trehalose biosynthesis pathways in other microbes. The results are summarized in Table 2.

A search based on the known E. coli trehalose biosynthesis genes identified ORF Rv3490 as a homologue of trehalose-6-phosphate synthases from E. coli (OtsA) and yeast (TPS1). A closely related ORF was found in the partially completed genome sequence of M. leprae. Two M. tuberculosis ORFs showed similarity to the phosphatase gene encoded by otsB in E. coli. Rv2006 encodes a protein with 1327 aa (named ots $B$ by Cole et al., 1998), and Rv3372 a smaller protein of 391 aa (otsB2). Both ORFs share a 260 aa 'core' region which is closely related to E. coli OtsB (266 aa) and to a similar core region of yeast TPS2 (896 aa). A related ORF in $M$. leprae encodes a 429 aa protein, again sharing the same core region. The different structures of the OtsB homologues are illustrated diagrammatically in Fig. 2. The additional $\mathrm{N}$-terminal and C-terminal domains of the M. tuberculosis otsB gene share homology with two other M. tuberculosis ORFs, Rv3400 (35\% identity over $238 \mathrm{aa}$ ) and $\operatorname{Rv} 3401$ (27\% identity over $777 \mathrm{aa}$ ), respectively. A more limited homology is observed with N-terminal domains of M. tuberculosis otsB2 (residues 190-282, 48\% identity) and M. leprae otsB (residues 
Table 2. Mycobacterial homologues of trehalose biosynthesis enzymes

\begin{tabular}{|c|c|c|c|c|c|c|c|}
\hline $\begin{array}{l}\text { M. tuberculosis } \\
\text { ORF }\end{array}$ & $\begin{array}{l}\text { Length } \\
\quad(\mathbf{a a})\end{array}$ & Name & Matching sequences & $\begin{array}{c}\text { Accession } \\
\text { no. }\end{array}$ & $\begin{array}{l}\text { Length } \\
\text { (aa) }\end{array}$ & $\begin{array}{c}\text { Identity } \\
(\%)\end{array}$ & $\begin{array}{c}\text { M. leprae } \\
\text { homologue } \\
\text { accession no. }\end{array}$ \\
\hline \multirow[t]{2}{*}{ Rv3490 } & \multirow[t]{2}{*}{500} & \multirow[t]{2}{*}{ OtsA } & E. coli OtsA & P31677 & 495 & 33 & \multirow[t]{2}{*}{ Q50167 } \\
\hline & & & Sac. cerevisiae TPS1 & Q00764 & 473 & 36 & \\
\hline \multirow[t]{2}{*}{ Rv2006 } & \multirow[t]{2}{*}{$1327^{*}$} & \multirow[t]{2}{*}{ OtsB } & E. coli OtsB & P31678 & 266 & 29 & \multirow[t]{2}{*}{ Q49734 } \\
\hline & & & Sac. cerevisiae TPS2 & P31688 & 896 & 28 & \\
\hline \multirow[t]{3}{*}{ Rv3372 } & \multirow[t]{3}{*}{391} & \multirow[t]{3}{*}{ OtsB2 } & E. coli OtsB & P31678 & 266 & 32 & \multirow[t]{3}{*}{ Q49734 } \\
\hline & & & Sac. cerevisiae TPS2 & P31688 & 896 & 25 & \\
\hline & & & M. tuberculosis OtsB & Rv2006 & 1327 & 57 & \\
\hline \multirow[t]{2}{*}{ Rv1563c } & \multirow[t]{2}{*}{765} & \multirow[t]{2}{*}{ TreY } & Rhizobium sp. & Q53237 & 772 & 45 & \multirow[t]{2}{*}{ Cosmid L458 } \\
\hline & & & Arthrobacter sp. & Q44315 & 775 & 46 & \\
\hline \multirow[t]{2}{*}{ Rv1562c } & \multirow[t]{2}{*}{580} & \multirow[t]{2}{*}{ TreZ } & Rhizobium sp. & Q53238 & 596 & 53 & \multirow[t]{2}{*}{ Cosmid L458 } \\
\hline & & & Arthrobacter sp. & Q44316 & 598 & 53 & \\
\hline \multirow[t]{2}{*}{ Rv0126 } & \multirow[t]{2}{*}{601} & \multirow[t]{2}{*}{ TreS } & Pimelobacter sp. & P72235 & 573 & 71 & \multirow[t]{2}{*}{ Cosmid B1610 } \\
\hline & & & Streptomyces sp. & O54207 & 572 & 69 & \\
\hline
\end{tabular}

*Identities listed are for the core region of OtsB from residue 283 to 532.

E. coli OtsB

Sac. cerevisiae TPS2

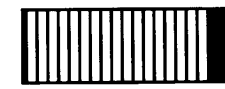

M. leprae OtsB

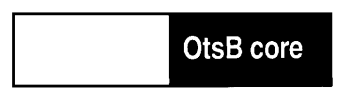
(Rv3372)

M. tuberculosis OtsB
(Rv2006)

M. tuberculosis
OtsB core

OtsB core

OtsB core

OtsB

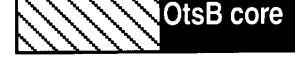

OtsB core
391 aa

266 aа

896 aa

429 aа

1327 aa

Fig. 2. OtsB homologues in mycobacteria. ORFs encoded by $M$. tuberculosis ots $B$ and ots $B 2$, and $M$. leprae ots $B$ share a conserved 260 aa core region closely related to $E$. coli otsB and S. cerevisiae TPS2. The additional N-terminal and Cterminal domains of the $M$. tuberculosis otsB gene share homology with two other $M$. tuberculosis ORFs, Rv3400 (35\% identity over $238 \mathrm{aa}$ ) and Rv3401 (27\% identity over $777 \mathrm{aa}$ ), respectively. A more limited homology is observed with Nterminal domains of $M$. tuberculosis otsB2 (residues 190-282, $48 \%$ identity) and $M$. leprae otsB (residues $81-160,28 \%$ identity).

81-160, $28 \%$ identity). ORFs Rv3400 and Rv3401 have been annotated by Cole et al. (1998) as a possible phosphoglucomutase and an unknown protein, respectively.

Two ORFs (Rv1563c and Rv1562c) were identified in the M. tuberculosis genome as having $45 \%$ and $53 \%$ overall identity at amino acid level with MOT synthase (TreY) and MOT trehalohydrolase (TreZ) from Rhizobium and Arthrobacter (Table 2). The genes have been named glgY and glgZ by Cole et al. (1998). We have used the designations tre $Y$ and tre $Z$ in this study, to reflect the close similarity to the genes in Rhizobium, Arthrobacter and Sulfolobus [also, glgY has previously been used as an alternative designation for glycogen phosphorylase, glgP, in E. coli (Rimmele \& Boos, 1994)]. The M. tuberculosis genes overlap by $7 \mathrm{bp}$, and are closely linked to a third gene, homologous to $g \lg X$ of $E$. coli and treX of Sulfolobus acidocaldarius, encoding an $\alpha$-amylase involved in glycogen debranching (Romeo et 
Table 3. Biosynthesis of trehalose in mycobacterial extracts

The functional activity of the three trehalose biosynthesis pathways was assayed by incubation of mycobacterial protein extracts $\left(0.5 \mathrm{mg}\right.$ protein $\left.\mathrm{ml}^{-1}\right)$ with appropriate substrates. Reactions were stopped at time points from 0-60 min, and glucose concentrations were measured before and after incubation with recombinant $E$. coli trehalase. The rate of trehalose biosynthesis was calculated by regression analysis over the linear phase of the reaction.

\begin{tabular}{|llcc|}
\hline Substrate & Pathway & \multicolumn{2}{c|}{ Trehalose generated $*$} \\
\cline { 3 - 4 } & & M. smegmatis & M. bovis BCG \\
\hline Glucose 6-phosphate & OtsAB & $9 \cdot 5$ & $5 \cdot 3$ \\
+ UDP-glucose & & & $18 \cdot 5$ \\
Maltoheptaose & TreYZ & $41 \cdot 6$ & $24 \cdot 4$ \\
Maltose & TreS & $14 \cdot 1$ & \\
\hline
\end{tabular}

*nmol trehalose $\min ^{-1}(\mathrm{mg} \text { protein })^{-1}$.

al., 1988). M. leprae cosmid L458 contains homologues of both tre $Y$ and treZ, although both are interrupted by frameshifts and stop codons.

Finally, ORF Rv0126 was found to share approximately $70 \%$ overall identity with trehalose synthase (TreS) from Pimelobacter (Tsusaki et al., 1996) and Streptomyces (Bruton et al., 1995). A similar ORF is present in M. leprae (Table 2).

\section{Analysis of enzyme function}

Analysis of the M. tuberculosis genome identified genes for three distinct trehalose-biosynthesis pathways. We then set out to determine whether these three pathways could be detected by functional assays based on enzyme activities using mycobacterial extracts and recombinant proteins expressed from the corresponding ORFs.

Trehalose from glucose monosaccharide. To measure activities of the OtsA-OtsB pathway, glucose 6-phosphate and UDP-glucose were incubated with cell-free extracts from $M$. smegmatis and $M$. bovis BCG. Formation of trehalose was detected by adding recombinant E. coli trehalase (TreA) and measuring the glucose produced over a $1 \mathrm{~h}$ incubation period. Trehalose synthesis was observed using both extracts (Table $3)$; there was no detectable synthesis in extracts incubated without substrate. Constant readings in samples that were not treated with trehalase showed that there was no detectable release of free glucose from the glucose 6-phosphate or UDP-glucose substrates during the incubation period. The optimum $\mathrm{pH}$ for the trehalose biosynthesis reaction was between pH 6 and 7 (Fig. 3).

To determine whether the ORFs identified by genome analysis were responsible for the biosynthetic activity observed in crude extracts, trehalose-6-phosphate synthase (OtsA) was purified from M. smegmatis using a minor modification of a procedure described by Pan et al. (1978). The N-terminal amino acid sequence of the purified protein, PESGHETISGTSDFVVVANRLPVzE-

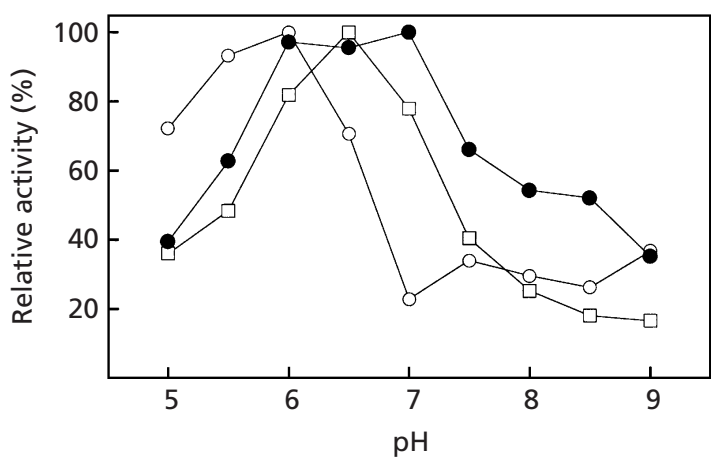

Fig. 3. Effect of $\mathrm{pH}$ on trehalose biosynthesis. Trehalose biosynthesis was measured in an $M$. smegmatis extract $(0.5 \mathrm{mg}$ protein $\mathrm{ml}^{-1}$ ) incubated with glucose 6-phosphate and UDPglucose (๑), maltoheptaose $(\square)$, or maltose (O). The $\mathrm{pH}$ of the reaction mixture was varied by changing the ratio of $\mathrm{Na}_{2} \mathrm{HPO}_{4}$ to $\mathrm{NaH}_{2} \mathrm{PO}_{4}$ in the assay buffer. Trehalose formation was measured as described in the legend to Table 3, and the percentage of the maximum activity is plotted for each pathway.

zLPDGQT, was identical to the M. tuberculosis OtsA ORF in 18 out of 30 residues, including an exact match over a stretch of 12 amino acids. This is consistent with the amino acid sequence of three internal peptides of the M. smegmatis enzyme determined by Pan et al. (1996), each of which showed over $75 \%$ identity to $M$. tuberculosis ots $A$. None of these peptide sequences produced comparable matches to other regions of the $M$. tuberculosis genomic sequence. Taken together, these results provide strong support for the conclusion that the M. tuberculosis otsA gene encodes the functional homologue of the trehalose-6-phosphate synthase activity detected in M. smegmatis. Direct attempts to measure activity of the M. tuberculosis gene product were frustrated by the fact that expression as a histidinetagged recombinant protein in E. coli resulted in the generation of insoluble protein lacking detectable enzyme activity. 
Mycobacterial sequences with OtsB homology - including the complete $\mathrm{N}$-terminal region and the central OtsB core domain - were also cloned in E. coli using the expression vector $\mathrm{pQE3} 3$ to generate proteins with an $\mathrm{N}$-terminal affinity tag suitable for rapid purification. Again, all of the constructs formed insoluble inclusion bodies when expression was induced by IPTG. These were solubilized by denaturation in $8 \mathrm{M}$ urea, purified by affinity chromatography, and then dialysed against decreasing concentrations of urea with the aim of allowing renaturation of functional enzymes. Activity was detected in one construct, which contained a 275 aa fragment from the M. leprae gene corresponding to the OtsB core domain. The purified renatured preparation catalysed the release of phosphate from trehalose 6phosphate, with specificity demonstrated by lack of activity when glucose 6-phosphate, galactose 6-phosphate or fructose 6-phosphate was used as substrate (data not shown). Renaturation was inefficient, and several micrograms of protein were needed to detect a small amount of activity, with poor reproducibility between different batches of recombinant protein. These results suggest that the sequence homology with the OtsB core region is functionally significant, though they do not allow us to determine whether one or both of the M. tuberculosis homologues contributes to trehalose-6phosphate phosphatase activity in cell extracts.

Trehalose from $\alpha(\mathbf{1} \rightarrow \mathbf{4})$-linked glucose polymer. To test for the presence of a functional TreY-TreZ pathway, $M$. smegmatis and M. bovis BCG extracts were incubated with maltoheptaose as substrate. Maltoheptaose is a heptameric oligosaccharide composed of glucose residues connected by $\alpha(1 \rightarrow 4)$ linkages identical to those found in glycogen. Again, biosynthesis of trehalose was observed (Table 3). The reaction was optimal at $\mathrm{pH} 6.5$ (Fig. 3). Formation of trehalose from maltoheptaose could occur by formation of a terminal $\alpha(1 \rightarrow 1)$ linked intermediate with subsequent cleavage to release the disaccharide (the TreY-TreZ pathway), or by initial hydrolysis to generate maltose, followed by conversion to trehalose by the trehalose synthase activity described below. The assay system based on unfractionated cell extract does not distinguish between these two potential pathways.

With the aim of direct measurement of specific enzyme activities, the $M$. tuberculosis tre $Y$ and tre $Z$ homologues were cloned in E. coli using the pQE30 expression vector. Again, the products were insoluble, and no functional activity could be recovered following affinity purification. Soluble products were obtained, however, when the genes were cloned into a mycobacterial expression vector, pACE, in M. smegmatis (De Smet et al., 1999). In this system, the genes are placed under the control of an acetamidase promoter, with expression induced by addition of acetamide to cultures. Recombinant protein was not visible on Coomassie bluestained gels, but production of trehalose from maltoheptaose was measured in soluble extracts from recombinant cultures, using a concentration selected to dilute out the background activity associated with the

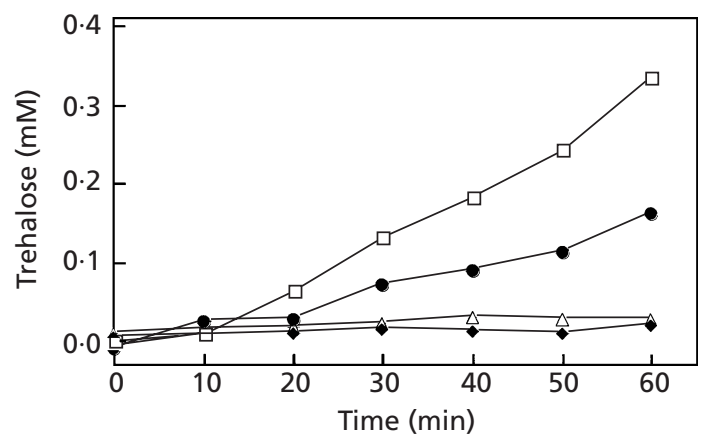

Fig. 4. Trehalose biosynthesis from maltoheptaose by recombinant mycobacterial TreY and TreZ enzymes. Biosynthesis of trehalose from maltoheptaose was assayed using extracts $\left(0.5 \mathrm{mg}\right.$ protein $\left.\mathrm{ml}^{-1}\right)$ prepared from recombinant $M$. smegmatis clones expressing the tre $Y$ and tre $Z$ genes of $M$. tuberculosis. Trehalose concentrations were calculated on the basis of glucose released following incubation with recombinant $E$. coli trehalase; results are corrected for free glucose observed in the absence of trehalase treatment. There was no detectable trehalose synthesis in the extract from vector control $M$. smegmatis $(\triangle)$ or in that from the TreY recombinant $(\diamond)$. Trehalose synthesis was observed using the extract from the TreZ recombinant (0), with enhanced activity when this was mixed with the TreY extract ( $\square$ ).

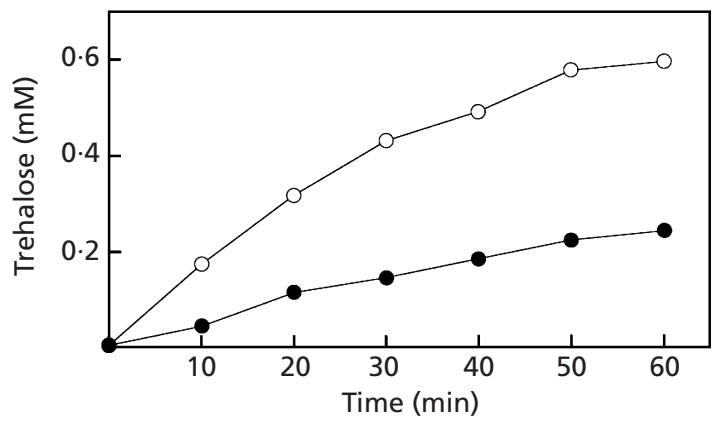

Fig. 5. Trehalose biosynthesis from maltose by recombinant mycobacterial TreS enzyme. Activity of the purified TreS recombinant is shown. The assay was carried out using maltose $(\bigcirc)$ or trehalose (O) as the substrate, and glucose was measured following treatment with trehalase or maltase respectively. Values are corrected for glucose detected prior to hydrolase treatment.

endogenous M. smegmatis enzymes in the vector-control extract (Fig. 4). There was no significant increase in trehalose synthesis using the extract from the recombinant expressing the M. tuberculosis MOT synthase (TreY) gene. Production of trehalose was observed, however, using the MOT trehalohydrolase (TreZ) recombinant, suggesting that the second step may be rate limiting in the pathway. This activity was enhanced when extract from the TreY recombinant was mixed with the TreZ extract. Enhanced activity was not observed if the TreY extract was replaced by the same concentration of the corresponding extract from the vector control. Fig. 4 shows results in trehalase-treated 
samples, corrected for background levels measured without trehalase. Direct release of free glucose was detected when extracts from the TreZ recombinant were incubated with maltoheptaose, but this activity was reduced following addition of the TreY extract. This suggests that the TreZ enzyme has a preference for hydrolysis of maltooligosyltrehalose, but also has additional $\alpha$-glucosidase activity.

Trehalose from maltose. Incubation of maltose with extracts from M. smegmatis and M. bovis BCG also resulted in production of trehalose (Table 3 ). The assay had a $\mathrm{pH}$ optimum of 6 (Fig. 3). Expression of the $M$. tuberculosis treS homologue as a His-tagged recombinant in E. coli using pQE30 generated mainly inactive, insoluble protein, but sufficient protein remained soluble for detection of enzyme activity. Enzyme activity able to convert maltose to trehalose was present in recombinant but not vector-control extracts (data not shown). The soluble recombinant protein retained trehalose synthase activity following purification by nickel affinity chromatography. Fig. 5 illustrates activity of the purified recombinant TreS using either trehalose or maltose as substrate. Although production of trehalose from maltose was some $2 \cdot 5$-fold greater than that of maltose from trehalose, the recombinant enzyme was capable of catalysing the reaction in both directions.

\section{DISCUSSION}

Biochemical experiments support the prediction from genome analysis that there are three routes for the synthesis of trehalose in mycobacteria. M. tuberculosis is unusual amongst the microbial genomes completed to date in having multiple pathways for trehalose biosynthesis; other organisms rely on a single pathway, most commonly OtsA-OtsB. Even the presence of two parallel pathways for the synthesis of a single product appears to be a rare occurrence, and to our knowledge has only been described in detail for diaminopimelate synthesis (Wehrmann et al., 1998) and glutamate (Helling, 1998).

Interestingly, analysis of the partially completed genome sequence of $M$. leprae suggests that this closely related pathogen has dispensed with the TreY-TreZ pathway. Cosmid L458 contains a genomic region similar to that encoding TreY-TreZ in M. tuberculosis, but with regions homologous to the $M$. tuberculosis ORFs disrupted by multiple frame shifts and stop codons. Comparison of the genetic organization in the two genomes suggests that $M$. leprae has evolved a lifestyle in which it no longer requires a third trehalosebiosynthesis pathway, thus removing the selective pressure for retention of the functionally active tre $Y$ and tre $Z$ genes.

In addition to having multiple distinct pathways for trehalose biosynthesis, M. tuberculosis also has two possible routes through the OtsA-OtsB pathway, with genome analysis identifying two enzymes containing the core functional domain of trehalose-6-phosphate phosphatase (OtsB). Both of the otsB genes are larger than their E. coli counterpart. Both have an additional Nterminal domain. In the case of the larger $M$. $t u$ berculosis OtsB, the $\mathrm{N}$-terminal domain resembles ORF Rv3400, encoding a potential phosphoglucomutase, while the additional C-terminal domain has sequence similarity to a protein of unknown function encoded by Rv3401. Thus the structure of the M. tuberculosis otsB gene appears to combine sequences related to two other ORFs that are adjacent in the genome $(\operatorname{Rv} 3400$ and Rv3401) fused together by insertion of a copy of the OtsB core region. Biochemical studies carried out so far have addressed the functional activity only of the OtsB core; it will be of interest to determine whether $M$. tuberculosis OtsB is in fact a multifunctional enzyme encoded as a single polypeptide. The significance of having two OtsB enzymes is unknown. It can be reasoned that the $\mathrm{N}$-terminal domain with potential phosphoglucomutase activity could facilitate trehalose biosynthesis by provision of the glucose 6-phosphate substrate, but the function of the C-terminal domain in the larger OtsB protein is unclear. Findings from genome-sequencing programmes indicate that the combination of multiple enzymes from a metabolic pathway within a single multifunctional polypeptide may be a relatively common phenomenon (Marcotta et al., 1999).

Accumulation of trehalose in E. coli and Sac. cerevisiae under stress conditions is thought to contribute to maintenance of cell integrity and protein stability (Strom \& Kaasen, 1993; De Virgilio et al., 1994). The constitutive presence of trehalose in apparently unstressed mycobacteria may likewise have a protective function. Trehalose may act as an osmoprotectant, for example (Poolman \& Glaasker, 1998). Consistent with such a role, the concentration of free trehalose relative to total protein increased 5-10-fold during growth of M. smegmatis in hyperosmotic medium (K. A. L. De Smet, unpublished observations). Bacteria are able to utilize a range of solutes to allow them to maintain their cytoplasmic volume and turgor pressure during exposure to osmotic stress (Csonka \& Hanson, 1991), and analysis of the $M$. tuberculosis genome reveals the presence of additional systems that could modulate solute transport in response to osmotic stress. These include homologues of the glycine-betaine transporter genes of Corynebacterium glutamicum (betP, Rv0917) and Bacillus subtilis (proW, proZ, proV and proX; Rv3756c, Rv3757c, Rv3758c and Rv3579c), and the trkA potassium transporter of Streptomyces coelicolor (Rv2691, Rv2692). However, the ability to regulate trehalose concentrations may have a particular advantage in allowing response to changing osmotic pressure while avoiding the need for transport of solutes through the relatively impermeable mycobacterial cell wall.

If trehalose is indeed required for maintenance of mycobacterial cell integrity in response to external stress, disruption of trehalose metabolism could provide a target for drugs which will act against both actively dividing cells and quiescent or non-dividing populations. It will be of interest to exploit recent refinements in 
techniques for mutagenesis of mycobacteria (Bardarov et al., 1997; Pelicic et al., 1998) to construct strains with disruptions in the genes described in the present study. While it might be anticipated that mutations will be compensated in part by complementary pathways, mutants might display unusual phenotypes under particular growth conditions which could provide insights into the basic function of trehalose in mycobacterial physiology.

\section{ACKNOWLEDGEMENTS}

Thanks to David Monsey for help with HPLC and Michael Ehrmann (University of Konstanz, Germany) for providing the $E$. coli clone expressing the recombinant trehalase, as well as a protocol to obtain periplasmic trehalase. The assistance of Peter Tormay in data analysis is also gratefully acknowledged. This work was funded by the Glaxo Wellcome Action TB Initiative.

\section{REFERENCES}

Bardarov, S., Kriakov, J., Carriere, C., Yu, S., Vaamonde, C., McAdam, R., Bloom, B., Hatfull, G. \& Jacobs, W. J. (1997). Conditionally replicating mycobacteriophages: a system for transposon delivery to Mycobacterium tuberculosis. Proc Natl Acad Sci USA 94, 10961-10966.

Belisle, J. T., Vissa, V. D., Sievert, T., Takayama, K., Brennan, P. J. \& Besra, G. S. (1997). Role of the major antigen of Mycobacterium tuberculosis in cell wall biogenesis. Science 276, 1420-1422.

Besra, G. S. \& Chatterjee, D. (1994). Lipids and carbohydrates of Mycobacterium tuberculosis. In Tuberculosis: Pathogenesis, Protection and Control, pp. 285-306. Edited by B. R. Bloom. Washington, DC: American Society for Microbiology.

Bruton, C. J., Plaskitt, K. A. \& Chater, K. F. (1995). Tissue-specific glycogen branching isoenzymes in a multicellular prokaryote, Streptomyces coelicolor A3(2). Mol Microbiol 18, 89-99.

Cole, S., Brosch, R., Parkhill, J. \& 39 other authors (1998). Deciphering the biology of Mycobacterium tuberculosis from the complete genome sequence. Nature 393, 537-544.

Csonka, L. N. \& Hanson, A. D. (1991). Prokaryotic osmoregulation: genetics and physiology. Annu Rev Microbiol 45, 569-606.

De Smet, K. A. L., Kempsell, K. E., Gallagher, A., Duncan, K. \& Young, D. B. (1999). Alteration of a single amino acid residue reverses fosfomycin resistance of recombinant MurA from Mycobacterium tuberculosis. Microbiology 145, 3177-3184.

De Virgilio, C., Hottiger, T., Dominguez, J., Boller, T. \& Wiemken, A. (1994). The role of trehalose synthesis for the acquisition of thermotolerance in yeast. I. Genetic evidence that trehalose is a thermoprotectant. Eur J Biochem 219, 179-186.

Elbein, A. D. \& Mitchell, M. (1973). Levels of glycogen and trehalose in Mycobacterium smegmatis and the purification and properties of the glycogen synthetase. J Bacteriol 113, 863-873.

Helling, R. B. (1998). Pathway choice in glutamate synthesis in Escherichia coli. J Bacteriol 180, 4571-4575.

Kaasen, I., Falkenberg, P., Styrvold, O. B. \& Strom, A. R. (1992). Molecular cloning and physical mapping of the otsBA genes, which encode the osmoregulatory trehalose pathway of Escherichia coli: evidence that transcription is activated by katF (AppR). J Bacteriol 174, 889-898 (published erratum appears in J Bacteriol 174, 3422).
Kaasen, I., McDougall, J. \& Strom, A. R. (1994). Analysis of the otsBA operon for osmoregulatory trehalose synthesis in Escherichia coli and homology of the OtsA and OtsB proteins to the yeast trehalose-6-phosphate synthase/phosphatase complex. Gene 145, 9-15.

Marcotta, E. M., Pellegrini, M., Ng, H.-L., Rice, D. W., Yeates, T. O. \& Eisenberg, D. (1999). Detecting protein function and proteinprotein interactions from genome sequences. Science 285, 751-753.

Maruta, K., Hattori, K., Nakada, T., Kubota, M., Sugimoto, T. \& Kurimoto, M. (1996a). Cloning and sequencing of trehalose biosynthesis genes from Arthrobacter sp. Q36. Biochim Biophys Acta 1289, 10-13.

Maruta, K., Hattori, K., Nakada, T., Kubota, M., Sugimoto, T. \& Kurimoto, M. (1996b). Cloning and sequencing of trehalose biosynthesis genes from Rhizobium sp. M-11. Biosci Biotechnol Biochem 60, 717-720.

Maruta, K., Mitsuzumi, H., Nakada, T., Kubota, M., Chaen, H., Fukuda, S., Sugimoto, T. \& Kurimoto, M. (1996c). Cloning and sequencing of a cluster of genes encoding novel enzymes of trehalose biosynthesis from thermophilic archaebacterium Sulfolobus acidocaldarius. Biochim Biophys Acta 1291, 177-181.

Matula, M., Mitchell, M. \& Elbein, A. D. (1971). Partial purification and properties of a highly specific trehalose phosphate phosphatase from Mycobacterium smegmatis. J Bacteriol 107, 217-222.

Moxon, E. R. (1995). Whole genome sequencing of pathogens: a new era in microbiology. Trends Microbiol 3, 335-337.

Nakada, T., Maruta, K., Tsusaki, K., Kubota, M., Chaen, H., Sugimoto, T., Kurimoto, M. \& Tsujisaka, Y. (1995). Purification and properties of a novel enzyme, maltooligosyl trehalose synthase, from Arthrobacter sp. Q36. Biosci Biotechnol Biochem 59, 2210-2214.

Nishimoto, T., Nakano, M., Nakada, T., Chaen, H., Fukuda, S., Sugimoto, T., Kurimoto, M. \& Tsujisaka, Y. (1996). Purification and properties of a novel enzyme, trehalose synthase, from Pimelobacter sp. R48. Biosci Biotechnol Biochem 60, 640-644.

Nossal, N. G. \& Heppel, L. A. (1966). The release of enzymes by osmotic shock from Escherichia coli in exponential phase. J Biol Chem 241, 3055-3062.

Pablos-Mendez, A., Raviglione, M. C., Laszlo, A. \& 8 other authors (1998). Global surveillance for antituberculosis-drug resistance, 1994-1997. World Health Organization-International Union against Tuberculosis and Lung Disease Working Group on AntiTuberculosis Drug Resistance Surveillance. N Engl J Med 338, 1641-1649.

Pan, Y. T., Mitchell, M. \& Elbein, A. D. (1978). Studies on the trehalose-phosphate synthase of Mycobacterium smegmatis: binding of heparin to the enzyme. Arch Biochem Biophys 186, 392-400.

Pan Y. T., Drake R. R. \& Elbein, A. D. (1996). Trehalose-P synthase of mycobacteria: its substrate specificity is affected by polyanions. Glycobiology 6, 453-461.

Parish, T. \& Stoker, N. G. (1997). Development and use of a conditional antisense mutagenesis system in mycobacteria. FEMS Microbiol Lett 154, 151-157.

Pelicic, V., Reyrat, J. M. \& Gicquel, B. (1998). Genetic advances for studying Mycobacterium tuberculosis pathogenicity. Mol Microbiol 28, 413-420.

Poolman, B. \& Glaasker, E. (1998). Regulation of compatible solute accumulation in bacteria. Mol Microbiol 29, 397-407.

Prinz, W. A., Spiess, C., Ehrmann, M., Schierle, C. \& Beckwith, J. 
(1996). Targeting of signal sequenceless proteins for export in Escherichia coli with altered protein translocase. EMBO J 15, 5209-5217.

Ranade, N. \& Vining, L. C. (1993). Accumulation of intracellular carbon reserves in relation to chloramphenicol biosynthesis by Streptomyces venezuelae. Can J Microbiol 39, 377-383.

Reinders, A., Burckert, N., Hohmann, S., Thevelein, J. M., Boller, T., Wiemken, A. \& De Virgilio, C. (1997). Structural analysis of the subunits of the trehalose-6-phosphate synthase/phosphatase complex in Saccharomyces cerevisiae and their function during heat shock. Mol Microbiol 24, 687-695.

Rimmele, M. \& Boos, W. (1994). Trehalose-6-phosphate hydrolase of Escherichia coli. J Bacteriol 176, 5654-5664.

Romeo, T., Kumar, A. \& Preiss, J. (1988). Analysis of the Escherichia coli glycogen gene cluster suggests that catabolic enzymes are encoded among the biosynthetic genes. Gene 70, 363-376.

Strom, A. R. \& Kaasen, I. (1993). Trehalose metabolism in Escherichia coli: stress protection and stress regulation of gene expression. Mol Microbiol 8, 205-210.
Tsusaki, K., Nishimoto, T., Nakada, T., Kubota, M., Chaen, H., Sugimoto, T. \& Kurimoto, M. (1996). Cloning and sequencing of trehalose synthase gene from Pimelobacter sp. R48. Biochim Biophys Acta 1290, 1-3.

Tsusaki, K., Nishimoto, T., Nakada, T., Kubota, M., Chaen, H., Fukuda, S., Sugimoto, T. \& Kurimoto, M. (1997). Cloning and sequencing of trehalose synthase gene from Thermus aquaticus ATCC 33923. Biochim Biophys Acta 1334, 28-32.

Wehrmann, A., Phillipp, B., Sahm, H. \& Eggeling, L. (1998). Different modes of diaminopimelate synthesis and their role in cell wall integrity: a study with Corynebacterium glutamicum. J Bacteriol 180, 3159-3165.

Winder, F. G., Tighe, J. J. \& Brennan, P. J. (1972). Turnover of acylglucose, acyltrehalose and free trehalose during growth of Mycobacterium smegmatis on glucose. J Gen Microbiol 73, 539-546.

Received 10 September 1999; revised 1 October 1999; accepted 5 October 1999. 